\title{
Interlocking Medullary Nail Versus Plate Fixation in Management of Diaphyseal Humeral Fracture
}

\author{
Omar Ab-Wahab Kelany ${ }^{1}$, Waleed Mohammad Nafae', Sameh Mohammad Holeil ${ }^{1}$, Aymen Faraj Saleh ${ }^{2}$ \\ ${ }^{1}$ Department of Orthopedic Surgery, Faculty of Medicine - Zagazig University, Sharkia, Egypt \\ ${ }^{2}$ Department of Orthopedic Surgery, Faculty of Medicine - Tripoli University, Tripoli, Libya \\ *Corresponding Author: Aymen Faraj Saleh, Email: abokalishaymenfarajyahoo@gmail.com, Mobile: (+20)01030499288
}

\begin{abstract}
Background: Humeral shaft fractures represent 3\% of all fractures, a significant number of these fractures require operative intervention. Objective: To compare the results of plate osteosynthesis and interlocking nailing in the treatment of diaphyseal humeral fracture concerning rate healing, functional outcome, and complications. Patients and Methods: This study is a prospective comparative study that included 18 patients in the period from December 2018 to July 2019 with follow up 8 months were operated up on at the Orthopedic Surgery Department of Zagazig University Hospitals. All patients had closed fractures in both groups. The commonest mechanism of injury was road traffic accidents (RTA) in 7 patients were in group I, 4 patients in group II. Falling from height in 3 patients were in group I and 4 patients were in group II. 9 cases of them were fixed by plates (group 1) and the other 9 by IM nails (group 2). Results: The results obtained were excellent in 15 patients (83.3\%) 6 patient fixed with a plate (66.6\%) and 9 patient fixed with nail (100\%), good in 2 patients (11.1\%) fixed with plate and Fair in one patient (5.5\%) fixed with a plate. The excellent and good results were considered satisfactory while fair and poor results were considered unsatisfactory. Conclusions: Plate fixation offered a higher likelihood of anatomic reduction of the fractures and predictable healing results, provided AO principles were followed. Although not quantifiable, it was our impression that antegrade nails were advantageous in comminuted AO Type $\mathrm{C}$ fractures.
\end{abstract}

Keywords: Shaft fractures, IM nails, Plate fixation.

\section{INTRODUCTION}

Humeral shaft fractures make up approximately $3 \%$ of all fractures. Typically, they are the result of direct trauma but also occur in sports where rotational forces are greater, for example, baseball or arm wrestling, Fractures of the middle or distal third of the shaft put the radial nerve at risk. In a small percentage of cases, humeral shaft fractures are associated with a vascular injury. Open fractures are uncommon but can represent serious injuries particularly if associated with crushing in industrial injuries ${ }^{(\mathbf{1})}$.

AO/ASIF classification humerus fractures are divided into three types with further subdivisions, A simple fracture, A1 - spiral fracture, A2 - oblique fracture, A3 - transverse fracture, B - wedge fractures, B1 - spiral wedge, B2 - bending wedge, B3 fragmented wedge, C- complex fractures, C1 complex spiral, C2 complex segmental fractures, C3 complex irregular fractures ${ }^{(2)}$.

Operative treatment for humerus fractures has usually been reserved for the treatment of non-union, unacceptable reduction of fractures, compound fractures, associated with forearm fractures, for polytrauma patients, fractures with neurovascular complications and patients with obesity who are at risk of developing varus angulations ${ }^{(3)}$.

There are currently three main operative techniques for definitively treating displaced humeral shaft fractures, open reduction with plate osteosynthesis (ORPO), intramedullary nailing $(\mathrm{IMN})$, and the more recently described minimally invasive plate osteosynthesis (MIPO). The use of the relative stability principle in diaphyseal fractures with minimally invasive techniques has gained interest for its potential advantage in causing few soft-tissue complications. Although the infection and nonunion rates do not differ for IMN and ORPO, there are other potential benefits of using minimally invasive techniques for humeral fractures: there is less need for bone grafts, there is a smaller incidence of radial nerve lesions, and there are less demanding techniques for complex or comminuted fractures. However, IMN has been how two cause more complications than ORPO, with a higher risk of shoulder pain and reoperation. The MIPO technique for humeral fractures has recently been described and was developed to allow the theoretical benefits of less soft tissue disruption without the complications of IMN ${ }^{(4)}$.

The present both of these surgical approaches are used to treat humeral shaft fractures. Both techniques have certain mechanical and anatomical advantages and disadvantages. Plating with stable fixation and direct visualization, which is known to provide an accurate anatomic reduction and protection of the radial nerve, can reduce the risk of malunion but requires wide intraoperative exposure associated with soft-tissue stripping. Continuous innovation in the design of IMN has ensured the clinical application of intramedullary fixation in treating humeral shaft fractures. Several studies recommended IMN as a standard surgical method through antegrade or 
retrograde nailing IMN has the advantage of closed insertion techniques, intact periosteal blood supply, and load-sharing mechanical properties. The IMN can reduce the effects of stress shielding at the fracture site and lower the incidence of refracture after implant removal. One primary complication of antegrade IMN is rotator cuff impairment, which might lead to shoulder impingement and the restriction of shoulder motion. Iatrogenic comminution of the fracture site during retrograde reaming and iatrogenic damage of the radial nerve during antegrade nailing are common complications during the operation ${ }^{(5)}$.

The most frequently reported complication after plate fixation of humeral shaft fractures is radial nerve palsy. When using an anterolateral (brachialissplitting) approach, it is essential to ensure that the nerve is not under the implant during plate application to avoid iatrogenic radial nerve injury. Posteriorly, soft tissue on the radial nerve can lead to iatrogenic injury in posterior approaches. This can be remedied by adequate soft tissue release of the radial nerve. Infection is reported to occur $1 \%$ to $2 \%$ of closed humeral fractures and $5 \%$ of open fractures occur in approximately $1 \%$ of patients. Nonunion of humeral shaft fractures is infrequent ${ }^{(6)}$.

\section{AIM OF THE WORK}

To compare the results of plate osteosynthesis and interlocking nailing in the treatment of diaphyseal humeral fracture regarding rate healing, functional outcome, and complications.

\section{PATIENTS AND METHODS}

This study is a prospective comparative study that included 18 patients in the period from December 2018 to July 2019 with follow up 8 months were operated up on at the Orthopedic Surgery Department of Zagazig University Hospitals. All patients had closed fractures in both groups. (group 1) 9 cases of them were fixed by plates and (group 2) 9 by IM nails.

\section{Ethical approval:}

Approval for performing the study was obtained from orthopedic surgery Departments, Zagazig University Hospitals after taking Institutional Review Board (IRB) approval, and also informed written consent was taken from patients and/or their caregivers.

This Work was performed according to the code of Ethics of the World Medical Association (Declaration of Helsinki) for studies involving humans.

They were all evaluated both clinically and radiologically. The clinical results were assessed according to the Constant-Murley shoulder scoring system and Stewart and Hundley's elbow criteria. Radiological evaluation included assessment of union, malunion, failure of fixation, and implant failure.
Inclusion criteria: Diaphyseal humeral fracture that required operative intervention and was treated with interlocking or plating procedures. Patients age 18 years or older. Comminuted diaphyseal humeral fracture.

Exclusion criteria: Patients age less than 18 years. Pathological fractures. Neurovascular injury. History of previous humeral fractures. Grade II and III open fractures. Fractures older than 2 weeks. A full workup including history taking, clinical examination, and radiological evaluation as well as laboratory investigations was performed for every patient on admission.

All patients underwent the surgical procedure under general anesthesia as soon as possible for every case according to general condition, time of operation was done from 1 to 5 days after injury.

\section{Operative Technique:}

Antegrade nailing group: Radiographs of the humerus were carefully studied. The fracture was examined for the degree of comminution and displacement. The width of the isthmus was measured. The isthmus of the humerus was usually located at the junction between the middle third and the distal third of the medullary canal. This measurement gave an idea about the expected diameter of the nail to be used and the need for reaming.

Anesthesia: The operation was performed under general anesthesia. All of our patients received intravenous $3^{\text {rd }}$ generation cephalosporin 30 minutes before the skin incision.

Patient positioning: We used a standard operating table with the radiolucent side table. The patient was placed diagonally on the operating table with the injured arm, abducted $45^{\circ}$, and resting on the radiolucent side table. The head part of the table was raised $30^{\circ}-40^{\circ}$ and the sandbag was placed under the scapula of the affected side, and the patient's face was turned towards the other side. The surgeon stood at the head of the patient. The assisting nurse stood beside him with the instrument trays. The assisting surgeon stood opposing to the surgeon, with the image intensifier between him and the nurse. This position ensured minimal movement of the personnel, at the same time ensured easy access during nailing and locking.

\section{Postoperative care:}

Every patient was put in an arm sling immediately post-operatively. Every patient was examined for vascular and neurological status. Check $\mathrm{X}$-rays were obtained to assess the reduction and the position of nails. Analgesic (parenteral $50 \mathrm{mg}$ of diclofenac sodium) twice daily and anti-oedematous (alpha chymotrypsin i.m. twice daily) measures were prescribed according to the clinical condition. One gram of third-generation cephalosporin was administered every twelve hours for 48 hours 
postoperatively. The postoperative dressing changed after 2 days.

\section{Follow up:}

Stitches were removed after two weeks. Rotational stresses were avoided until callus formation was visible. After two to three days the patient was instructed to remove the sling several times per day and start a passive range of motion exercises of the elbow and shoulder as tolerated by pain. The arm sling was removed after three to six weeks and active shoulder exercises were allowed. Heavy weight loading not allowed till complete and solid radiological bone union was achieved. Check Xrays (anteroposterior and lateral views) were obtained after two, six, and twelve weeks, then monthly till radiological union, then at the end of follow up. The mean follows up period was twenty-four weeks (range: from twenty to thirty-two).

\section{Complications:}

1. Nail protrusion: Nail protrusion into the shoulder was a technical error due to incomplete insertion of the nail as it should be a $2 \mathrm{~mm}$ subchondral. It occurred in one patient but the patient refused any operative procedure to remove the nail after the union.

2. Nerve injury: There was good result no nerve injury case.

3. Shoulder impingement: Shoulder impingement was seen in three patients' nail protrusion was the cause in one of them.
4. Infection: There were two patients with superficial infection in group 1 and one in group 2 undergo daily dressing and third-generation cephalosporin until improvement, no statistically significant relation between group1 and group 2 in the final score.

5. Limitation of shoulder and elbow motion: Shoulder range of motion was full in plate group and one patient fair and two patients good and six patients full in nail group. elbow range of motion was full in plate group and one patient fair and two patients good and six patients full in nail group.

6. Non-union: There was a good result no non-union cases all the patients union at six months.

\section{Statistical analysis}

An independent t-test was conducted to compare continuous variables and the chi-square and Fisher's exact tests were performed to compare categorical variables. Continuous variables are expressed as mean \pm standard deviation and categorical variables as frequency and percentage. A multivariate linear regression analysis was conducted to determine the independent correlation between the average aortic valve sclerosis score index and other study variables. $P$ values less than 0.05 were considered significant. We performed all statistical analyses using the Statistical Package for the Social Sciences version 18 (SPSS, Inc., Chicago, IL, USA).

\section{CASES PRESENTATION}

Case (1):

Male patient admitted to the hospital with a history of a road traffic accident with fracture mid-shaft right humerus. AO classification arrangement: 12-A1. The operation was done using LCP with a locked plate placed anteriorly.

\section{Preoperative:}

Age: 24 years old, Sex: male, Side of injury: Right

Mechanism of injury: RTA, AO Classification System: 12 A1, Time interval: one day.

Operative:

Operative time (min): 120min, Plate position: Anterior, Radiation time (sec): 120 second

Postoperative: Follow up (wk): 28 weeks, Union (wk): 12 weeks, Active elbow flex/ext:0-120 , (Figure 1a,b,c)

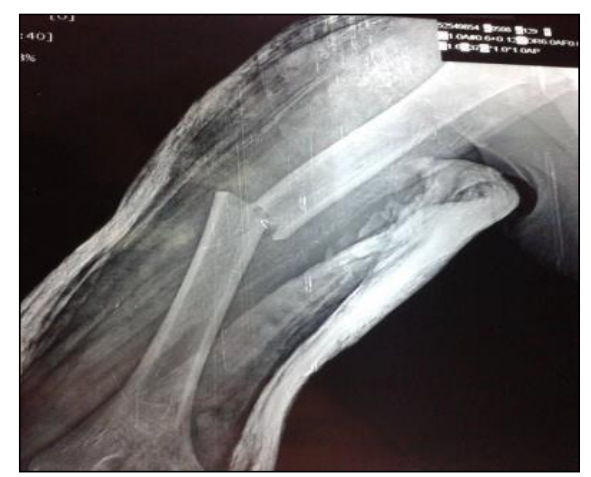

Figure (1a): Preoperative x-ray.

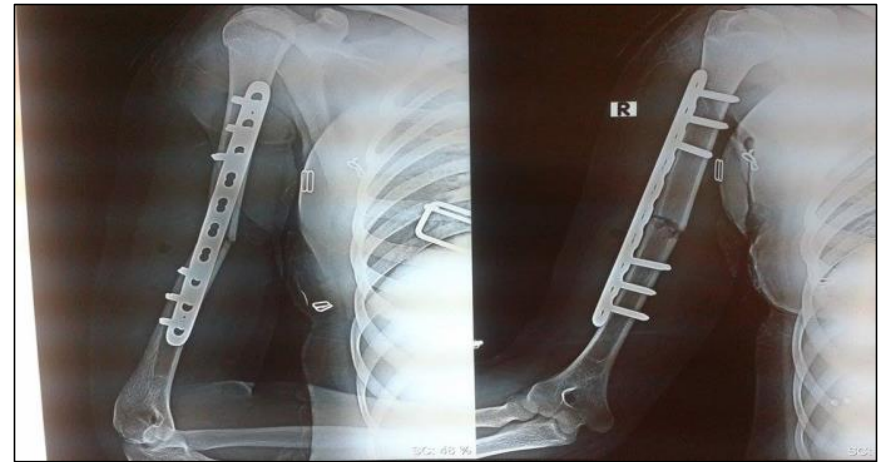

Figure (1b): Postoperative x-ray. 


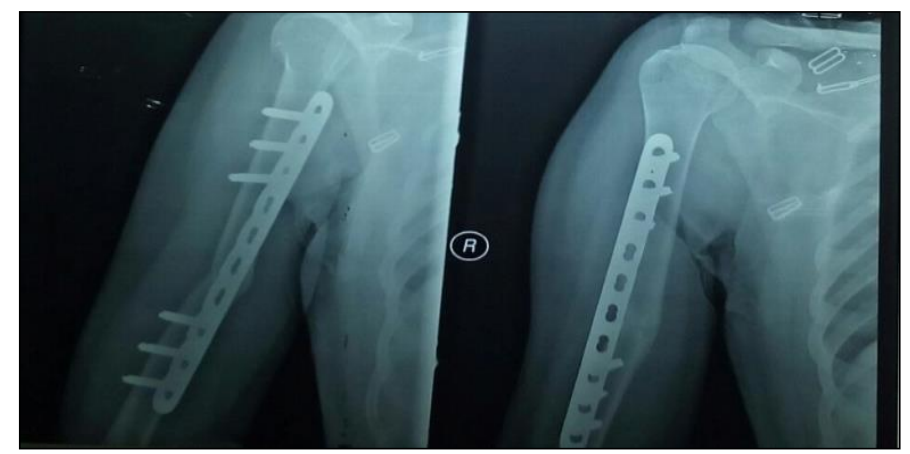

Figure (1c): 6 months follow up X-ray.

\section{CASE (2)}

Female patient 37years Old admitted to the hospital through a history of FH with fracture mid-shaft right humerus. AO classification system: 12-A1. The operation was done using antegrade nail humeral technique.

\section{Preoperative:}

Age: 37 years old, Sex: female, Side of injury: Right, Mechanism of injury: FH, AO

Classification System: 12- A1, Time interval: 1 days.

Operative: Operative time ( $\mathrm{min})$ : $90 \mathrm{~min}$, Radiation time (sec): 120 seconds.

Postoperative: Follow up (wk):28 weeks, Union (wk): 16 weeks, Active elbow flex/ext: 0 - 110 , (Figure 2a,b,c,d)

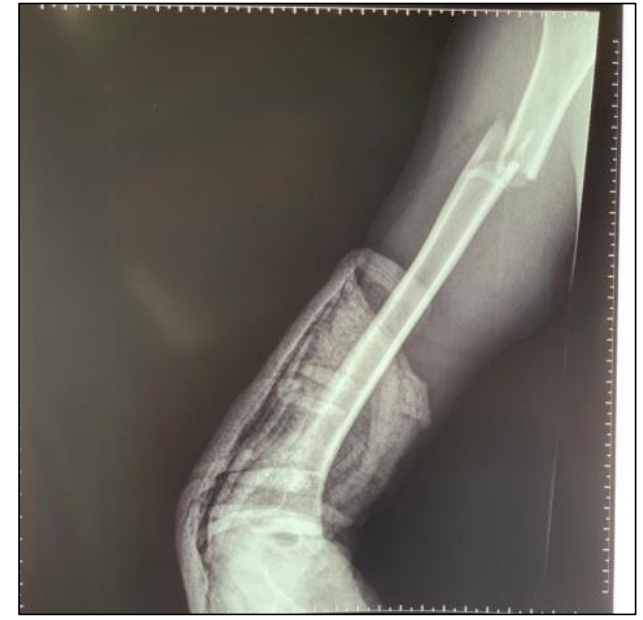

Figure (2a): Preoperative x-ray.

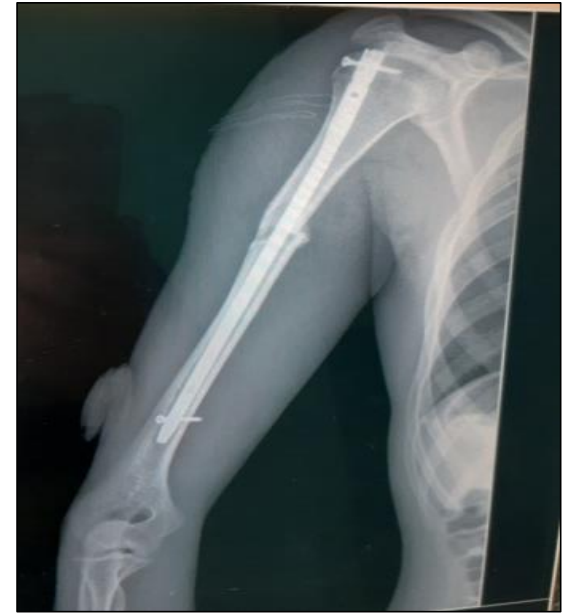

Figure (2b): Postoperative x-ray.

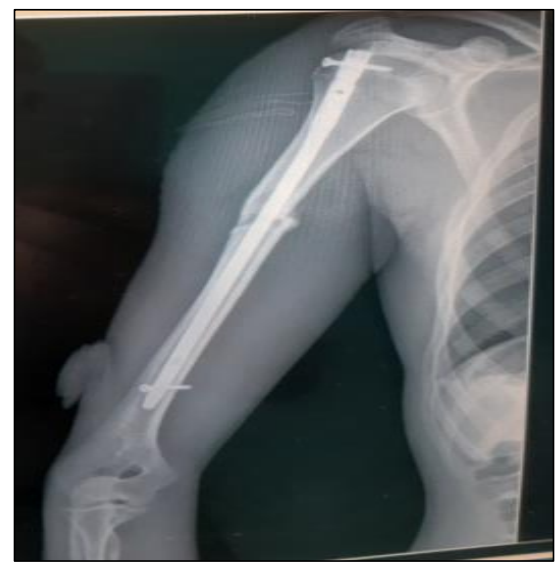

Figure (2c): Postoperative x-ray. 
RESULTS

Table (1): Socio-demographic data distribution among the studied group ( $N=18)$.

\begin{tabular}{|l|l|c|c|}
\hline \multicolumn{2}{|c|}{} & \multicolumn{2}{c|}{ Age/ Years } \\
\hline Mean \pm SD & \multicolumn{2}{c|}{$35.77 \pm 10.06$} \\
\hline \multirow{2}{*}{ Sex } & Female & N & $\%$ \\
\cline { 2 - 4 } & Male & 6 & 33.3 \\
\hline \multirow{3}{*}{ Heavy work } & No & 12 & 66.7 \\
\cline { 2 - 4 } & Yes & 11 & 38.9 \\
\cline { 2 - 4 } & Total & 18 & $\mathbf{6 1 . 1}$ \\
\hline
\end{tabular}

Age distributed as $35.77 \pm 10.06$ years with minimum 21 and a maximum 53, regarding sex males were $66.7 \%$ and female $33.3 \%$ and regarding occupation, $61.1 \%$ did heavy work and $38.9 \%$ didn't do heavy work.

Table (2): Comparison between the outcome of different management.

\begin{tabular}{|c|c|c|c|c|c|c|}
\hline \multicolumn{3}{|l|}{\begin{tabular}{|l} 
\\
\end{tabular}} & Nail group & Plate group & $t / \mathrm{X} 2$ & $\overline{\mathbf{P}}$ \\
\hline \multicolumn{3}{|l|}{ Time union } & $2.94 \pm 0.46$ & $3.5 \pm 0.93$ & -1.59 & 0.13 \\
\hline \multirow[t]{6}{*}{ Pain } & \multirow[t]{2}{*}{ Fair } & $\mathbf{N}$ & 1 & 3 & \multirow{6}{*}{2.91} & \multirow[t]{6}{*}{0.23} \\
\hline & & $\%$ & $11.1 \%$ & $33.3 \%$ & & \\
\hline & \multirow[t]{2}{*}{ Good } & $\mathbf{N}$ & 4 & 1 & & \\
\hline & & $\%$ & $44.4 \%$ & $11.1 \%$ & & \\
\hline & \multirow{2}{*}{ Excellent } & $\mathbf{N}$ & 4 & 5 & & \\
\hline & & $\%$ & $44.4 \%$ & $55.6 \%$ & & \\
\hline \multirow{6}{*}{$\begin{array}{l}\text { Shoulder } \\
\text { movement }\end{array}$} & \multirow[t]{2}{*}{ Fair } & $\mathbf{N}$ & $\mathbf{0}$ & 1 & \multirow[t]{6}{*}{3.6} & \multirow[t]{6}{*}{0.16} \\
\hline & & $\%$ & $0.0 \%$ & $11.1 \%$ & & \\
\hline & \multirow[t]{2}{*}{ Good } & $\mathbf{N}$ & $\mathbf{0}$ & 2 & & \\
\hline & & $\%$ & $0.0 \%$ & $22.2 \%$ & & \\
\hline & \multirow[t]{2}{*}{ Full } & $\mathbf{N}$ & 9 & 6 & & \\
\hline & & $\%$ & $100.0 \%$ & $66.7 \%$ & & \\
\hline \multirow{6}{*}{$\begin{array}{l}\text { Elbow } \\
\text { movement }\end{array}$} & \multirow[t]{2}{*}{ Fair } & $\mathbf{N}$ & 0 & 1 & \multirow[t]{6}{*}{3.6} & \multirow[t]{6}{*}{0.10} \\
\hline & & $\%$ & $0.0 \%$ & $11.1 \%$ & & \\
\hline & \multirow[t]{2}{*}{ Good } & $\mathbf{N}$ & $\mathbf{0}$ & 2 & & \\
\hline & & $\%$ & $0.0 \%$ & $22.2 \%$ & & \\
\hline & \multirow[t]{2}{*}{ Full } & $\mathbf{N}$ & 9 & 6 & & \\
\hline & & $\%$ & $100.0 \%$ & $66.7 \%$ & & \\
\hline \multirow[t]{4}{*}{ Infection } & \multirow[t]{2}{*}{ No } & $\mathbf{N}$ & 8 & 7 & \multirow[t]{4}{*}{0.4} & \multirow[t]{4}{*}{0.52} \\
\hline & & $\%$ & $88.9 \%$ & $\mathbf{7 7 . 8 \%}$ & & \\
\hline & \multirow[t]{2}{*}{ Yes } & $\mathbf{N}$ & 1 & 2 & & \\
\hline & & $\%$ & $11.1 \%$ & $22.2 \%$ & & \\
\hline \multirow[t]{6}{*}{ Outcome } & \multirow[t]{2}{*}{ Excellent } & $\mathbf{N}$ & 8 & 6 & \multirow[t]{6}{*}{2.28} & 0.31 \\
\hline & & $\%$ & $88.9 \%$ & $66.7 \%$ & & \\
\hline & Fair & $\mathbf{N}$ & $\mathbf{0}$ & 2 & & \\
\hline & & $\%$ & $0.0 \%$ & $22.2 \%$ & & \\
\hline & Good & $\mathbf{N}$ & 1 & 1 & & \\
\hline & & $\%$ & $11.1 \%$ & $11.1 \%$ & & \\
\hline Satisfaction & Satisfactory & $\mathbf{N}$ & 9 & 7 & 2.25 & 0.13 \\
\hline & & $\%$ & $100.0 \%$ & $\mathbf{7 7 . 8 \%}$ & & \\
\hline & Unsatisfactory & $\mathbf{N}$ & 0 & 2 & & \\
\hline & & $\%$ & $0.0 \%$ & $22.2 \%$ & & \\
\hline Total & & $\mathbf{N}$ & 9 & 9 & & \\
\hline & & $\%$ & $100.0 \%$ & $100.0 \%$ & & \\
\hline
\end{tabular}

There was no significant difference between the nail group and the plate group. Table 1 


\section{DISCUSSION \\ Union}

Most current operative methods for stabilization of humeral shaft fractures have acceptable rates of the union.

In this study, the fractures tend to unite an average of 8 to 14 weeks.

Naga and Somesula (7) the study subjects consisted of $n=28$ adult patients of fracture shaft of humerus, in plating group, $12(86.67 \%)$ patients recovered completely and $\mathrm{n}=3(20 \%)$ cases had complications. There was an incidence of postoperative radial nerve palsy and fully recovered following the use of neurotrophic drugs for 3 and 6 weeks after surgery. $1(6.67 \%)$ case had non-union as the patient lifted heavy weight leading to hypertrophic non-union and resulted in a poor result. The IMN group had no non-union, Abdallah et al. ${ }^{(8)}$ forty patients with humeral shaft fractures were selected randomly for treatment by either an antegrade interlocking nail or by a DCP plate and screws; after obtaining consent, 20 patients were included in each group three patients from the nail group had delayed union, the healing was delayed after 5 months in contrast to plate group, only one case had delayed union. Nonunion was recorded in three cases in the nail group that needed further active intervention; the incidence was $15 \%$. In contrast to the plate group, in only two cases there was nonunion and needed revision, Shobha et al. (9) included 20 patients operated with open reduction and internal fixation with locking compression plates and 20 patients operated with closed reduction and internal fixation with intramedullary nails. 1 patient in each group were found to have delayed union. They were closely followed up and their fractures eventually united the two groups had no cases non-union.

In this series, the rate of union compared well with these results as we had no cases of non-union.

\section{Infection:}

Because of the good vascular supply and large soft tissue sleeve surrounding the humerus, infection is relatively infrequent after the operative stabilization of humeral fractures and seems to be more common after open techniques of fixation.

In this series, there were 2 cases of superficial infection encountered in the 9 cases in Group I treated by debridement and antibiotic iv.

Abdallah et al. ${ }^{(8)}$ Forty patients with humeral shaft fractures were selected randomly for treatment by either an antegrade interlocking nail or by a DCP plate and screws two cases in the plate group had a superficial infection in the early postoperative period; in one of them, the fracture was an open grade two and in the second case, it was a closed type. Both cases after debridement infection subsided early incidence was $10 \%$. In contrast, in the nail group, no postoperative infection cases were recorded, $0 \%$.
Naga and Somesula (7) the study subjects consisted of $n=28$ adult patients of fracture shaft of the humerus with indications for surgical management In plating group, 1 (6.67\%) patient had wound infection and recovered with antibiotics and resulted in a good result, in the interlocking nailing group, 1 (7.69\%) subject had a superficial infection at the fracture site and later the wound healed well.

\section{Elbow function}

The functional outcome between two groups was assessed through Stewart and Hundley's scoring system 15 patients $(83.33 \%)$ had a full range of motion; 2 patients good in group I (11\%) had a loss of less than 20 degrees of elbow extension, one patient fair $(5.5 \%)$ had a loss of less than 40 degrees of elbow extension.

The lost degrees of elbow extension in group 1 patients may be explained by fibrosis that occur through triceps splitting affect extensor mechanism. This affects the full action of the triceps muscle.

Concluded that plate fixation offered an anatomic reduction of the fracture and predictable healing results.

Belayneh et al. ${ }^{(10)}$ conducted their study on 34 patients treated with open reduction and internal fixation. They found 6 patients had suffered from restriction in elbow extension after using the posterior approach as surgical exposure. This is similar to our study finding regarding the range of elbow function after the posterior approach and rate of non-union. explained rate postoperative restriction of elbow movement by excessive surgical dissection that affects the mechanism of triceps action.

\section{Shoulder Function:}

The most frequent criticism of antegrade humeral nailing has been its potentially deleterious effect on shoulder function.

In our study recommended an antegrade insertion point lateral and distal to the rotator cuff. In a series of 9 acute humeral fractures treated with antegrade nails, reported that one $(11 \%)$ patients had shoulder pain and decreased shoulder abduction because of impingement of the proximal locking screws. Removal of the proximal screws in one of these patients resulted in complete resolution of symptoms.

Shobha et al. ${ }^{(9)}$ included 20 patients operated with open reduction and internal fixation with locking compression plates and 20 patients operated with closed reduction and internal fixation with intramedullary nails was to achieve full range of movements by 8 to 12 weeks, but avoiding active external rotation beyond 40 degrees and forward flexion beyond 90 degrees. Between 6 to 12 weeks, the progression of a range of movements from the closed chain to an open-chain was done. After 12 weeks, strengthening exercises were advised to the patients in the form of light weights and band exercises, Excellent $>30 \%$ Full range of movements, Good $<10 \%$ average 
loss of movements, fair 10-30\% average loss of movements, Poor $>30 \%$ average loss of movements.

Sandhu et al. (11) in 30 cases 9 patients (20\%) 5 patients in a plate and 4 patients nail, 7 patients $(20-$ $40 \%$ ) 1 patient in a plate and 6 patients nail, 1 patient $(40 \%)$ in nail only, 13 patients full rang motion excellent 9 patients $(60 \%)$ plate and excellent 4(27\%) patients nail.

\section{CONCLUSION}

That the majority of humeral shaft fractures can be treated safely and effectively by non-operative methods. However, for the subset of patients requiring surgical treatment, intramedullary nailing, and plating both provide predictable means of achieving fracture stabilization and ultimate healing. In this series of patients, neither method was shown to be markedly superior to the other, although nails were associated with a higher incidence of shoulder discomfort. The selection of treatment for these fractures should be based on patient factors (e.g., age, sex, and occupation), fracture morphology, location, soft tissue injury, and surgeon preference. Either method, properly selected and performed, should lead to successful results in a large majority of patients.

\section{REFERENCES}

1. Richard EB, Christopher GM, Theerachai A (2018): Humerus Shaft Fractures. AO Principles of Fracture Management, Third Edition. https://www.amazon.com/AO-Principles-FractureManagement-fractures-ebook/dp/B0785JQ5XP

2. Kodandapani K, Kumar NR, Prasad KV (2016): Plating/Nailing the Ideal Fixation for Diaphyseal Fractures of Humerus-Our Experience. IOSR Journal of Medical Sciences, 15(9): 32-42.
3. Rathod J, Patil P, Kanake V, Kawalkar U (2017): Comparison between Interlock Nailing and Platting for Fracture Shaft Humerus. International Journal of Contemporary Medical Research, 4(3): 587-90.

4. Benegas E, Neto A, Gracitelli M et al. (2014): Shoulder function after surgical treatment of displaced fractures of the humeral shaft: a randomized trial comparing antegrade intramedullary nailing with minimally invasive plate osteosynthesis. Journal of Shoulder and Elbow Surgery, 23(6): 767-74.

5. Ma J, Xing D, Ma X et al. (2013): Intramedullary nail versus dynamic compression plate fixation in treating humeral shaft fractures: grading the evidence through a meta-analysis. PLoS One, 8(12): e82075.

6. Frederick M, Azar, James H, Beaty et al. (2017): Fractures of the Shoulder, Arm, and Forearm. Campbell's Operative Orthopaedics, Thirteenth edition, 4(57): 2953-7.

7. Naga V, Somesula J (2018): A comparative study on the fixation of fracture shaft of the humerus in adults by compression plate and intramedullary interlocking nail. International Journal of Orthopaedics, 4(1):31622.

8. Abdallah H, El din M, Rizk A et al. (2018): Results of compression plating versus locked intramedullary nailing fixation in the treatment of humeral shaft fractures in adults. Journal of Medicine in Scientific Research,1(3):214-18.

9. Shobha H, Kumar PS, Jolly A et al. (2017): Middle third humerus fractures in adults: Intramedullary nails vs. locking compression plates. International Journal of Orthopaedics Sciences, 3(4):742-5.

10. Belayneh R, Lott A, Haglin J et al. (2019): Outcomes of radial nerve palsy associated with humeral shaft fracture and nonunion. Journal of Orthopaedics and Traumatology, 20(1):18-22.

11. Sandhu KS, Bakshi AS, Banga RK et al. (2018): Functional outcomes in humeral shaft fracturesConservative management versus operative procedure. International Journal of Orthopaedics, 4(1): 243-46. 